\title{
Movement Recognition Technology Based Method for Evaluating Ability on Flight Operation Objectively
}

\author{
Chunmei Gui ${ }^{1, a,{ }^{*},}$ Xiaohong Shi ${ }^{2, b}$, Jian Qiang ${ }^{1, a}$, Wang Jie ${ }^{1, a}$, Zheng Wei ${ }^{1, a}$, Bai \\ $\mathrm{Yu}^{1, \mathrm{a}}$, and Du Jian ${ }^{1, \mathrm{a}}$ \\ ${ }^{1}$ Institute of Aviation Medicine, Air Force, Beijing, China \\ ${ }^{2}$ School of Computer Science, National University of Defense Technology, Changsha, Hunan \\ aplantsperfume@aliyun.com, bgcmjq2002@163.com \\ ${ }^{*}$ Chunmei Gui
}

\begin{abstract}
Movement recognition technology is based to evaluate ability on flight operation. By analyzing the changing characteristics of flight parameters in different flight actions, and making full use of the domain expert's prior knowledge, we can establish expert system reasoning machine for recognition knowledge base of flight action. First, the actual flight data is read in frame by frame, and reasoning machine will match each rule in the knowledge base. If flight rule of describing output matches successfully, the flight action name, completion time and related flight parameters would be output to the specified data file according to specified contents in the knowledge base.
\end{abstract}

Keywords: Ability on flight operation, Flight parameters, Movement recognition technology, rule matching, objective evaluation.

\author{
基于动作识别技术建立飞行操作能力客观评价方法

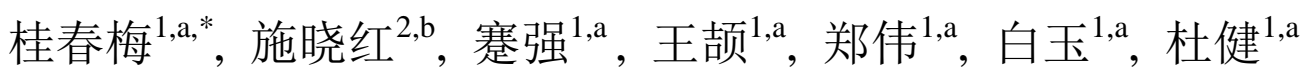 \\ 1空军航空医学研究所, 北京, 中国 \\ 2 国防科学技术大学计算机学院, 长沙, 中国 \\ aplantsperfume@aliyun.com, bgcmjq2002@163.com \\ *桂春梅
}

中文摘要. 基于飞行动作识别技术评估飞 行操作技能。分析不同飞行动作中的飞行参 数变化特征, 充分利用领域专家先验知识, 建立飞行动作识别知识库的专家系统推理 机, 逐帧读入实际的飞行数据, 推理机对知 识库中的每一条规则进行匹配。当描述输出
飞行动作的规则匹配成功时, 根据知识库指 定的内容, 将飞行动作名称、完成时间和相 关的飞行参数输出到指定的数据文件中。根 据评估指标与算法完成对飞行操作能力的 客观评价。 
关键词: 飞行操作能力; 飞行参数; 动作识 别技术; 规则匹配; 客观评价

\section{1. 引言}

本文针对飞行员飞行操作能力的训练 和测评需求, 建立仿真环境下飞行员飞行操 作能力测评方法 ${ }^{[1]}$, 旨在利用较低成本对飞 行员进行各项操作的能力测试, 从而提前发 现检测参试飞行员存在的不足。该方法可基 于网络环境, 结合任务背景进行开发、部署 和调试, 为飞行员虚拟任务为背景的操作训 练设备和环境构建提供核心技术。

\section{2. 飞行动作识别与评价}

首先开展飞行动作知识获取, 然后进行 飞行动作归类和特征分析, 接着建立飞行动 作识别知识库并用产生式规则 ${ }^{[2]}$ 加以描述, 最后利用飞行动作识别知识库推理识别仿 真条件下飞行动作, 并给出成绩评定。

\section{1 知识获取}

知识获取的任务就是将人类已有的知 识从领域专家的大脑中或书本上抽取出来, 加以归纳总结, 编码成计算机可以利用的形 式, 并输入到计算机中, 为计算机完成领域 专家所能的工作提供知识基础。

知识获取的方式可分为直接方式和间 接方式两种: 直接方式由领域专家向专家系 统提供一定数量的数据和资料, 应用统计归 纳、因果推理等技术从中提取出所需知识, 类似于数据库领域的数据挖掘过程; 间接方 式首先领域专家将自己的知识用语言及书 面的形式整理出来; 然后知识工程师在专家 的帮助下对所提供的知识进行分析、抽象及 简化, 编码成能被计算机理解的形式, 通过 知识编译器之类的工具, 将相关知识输入到 专家系统的知识库中。

本论文采用的知识获取主要采用间接 方式, 飞行动作特征由领域专家整理、由知 识工程师编码形成产生式规则。

\section{2 飞行动作特征分析}

战机在基本驾驶技术训练阶段常见的 飞行科目有仪表、简单特技和复杂特技等, 动作编排方式参见表1。对表1加以分析、归
纳, 可将飞行动作分为盘旋类、升降转弯类、 俯冲跃升类、横滚类、斤斗类和特殊飞行任 务类等6类，有些复杂的特技动作经过分解 后也可归为这几类, 如半斤斗翻转可以分解 为半斤斗和翻转, 半斤斗归属于斤斗类, 翻 转归属于横滚类。

经过对大量的实际飞行数据的综合分 析, 总结出在完成不同类型的飞行动作时, 其飞行参数的典型变化特征。各飞行动作变 化特征可各自分解归类, 例如, 升降转弯类 动作飞行参数变化特征如表1所示。

表1 升降转弯类动作飞行参数变化特征

\begin{tabular}{|c|c|c|c|}
\hline 飞行参数 & $\begin{array}{c}\text { 坡度 } 45^{\circ}, \\
\mathrm{V} 3=15 \mathrm{~m} / \mathrm{s} \\
\Delta \mathrm{H}=1000 \mathrm{~m} \\
\text { 升降转弯 }\end{array}$ & $\begin{array}{c}\text { 坡度 } 45^{\circ}, \\
\mathrm{V} 3=30 \mathrm{~m} / \mathrm{s} \\
\Delta \mathrm{H}=2000 \mathrm{~m} \\
\text { 升降转弯 }\end{array}$ & $\begin{array}{c}\text { 坡度 } 60^{\circ}, \\
\mathrm{V} 3=30 \mathrm{~m} / \mathrm{s} \\
\Delta \mathrm{H}=2000 \mathrm{~m} \\
\text { 升降转弯 }\end{array}$ \\
\hline 坡度/( ) & $\approx \pm 45$ & $\approx \pm 45$ & $\approx \pm 60$ \\
\hline $\begin{array}{c}\text { 下降时俯仰角 } \\
/\left(^{\circ}\right)\end{array}$ & $\approx-1.5$ & $\approx-7$ & $\approx-7$ \\
\hline $\begin{array}{c}\text { 上升时俯仰角 } \\
/\left(^{\circ}\right)\end{array}$ & $\approx 8$ & $\approx 13$ & $\approx 13$ \\
\hline $\begin{array}{c}\text { 航向累加变化量 } \\
/\left(^{\circ}\right)\end{array}$ & $\approx \pm 180$ & $\approx \pm 180$ & $\approx \pm 180$ \\
\hline $\begin{array}{c}\text { 速度累加变化量/ } \\
(\mathrm{km} \cdot \mathrm{h}-1)\end{array}$ & $< \pm 120$ & $< \pm 120$ & $< \pm 120$ \\
\hline
\end{tabular}

如果该项数值较小或变化不确定或可以 不予考虑, 表中所描述的各类动作飞行参数 的变化特征是建立飞行动作识别知识库的 基础。

\section{3 建立飞行动作识别知识库}

2.3.1 描述规则:

为了便于工程实现, 经过分析、论证, 飞行动作识别和知识库采用产生式规则加 以描述。

产生式规则的一般表达形式为:

IF A THEN B f(B,A)

$f(B, A)$ 为当条件 $A$ 成立时, 结果 $B$ 成立的 可能性。

在具体实现过程中, 采用三行语句描 述:

条件行: 事件代号、成立条件、持续时 间、事件性质、事件名称;

操作行: 事件成立后所要进行的操作;

输出行: 事件成立后输出的内容。

在飞行动作识别知识库中, 每一种动作 都可以用这种知识表达形式加以描述。下面 
以坡度 $45^{\circ}$ 盘旋动作为例, 说明动作识别知 识库的建立过程。

\subsection{2 建立过程}

1. 设立坡度 $45^{\circ}$ 盘旋起始标志。

1) 符号定义:

BZpxdz : 盘旋起始标志;

TPGama2：倾斜角变化量绝对值;

$\begin{array}{ll}\text { ! } & \text { : 逻辑 “非”; } \\ \| & \text { : 逻辑 “或”; } \\ \text { Gama } & \text { : 倾斜角; } \\ \text { Sita } & \text { : 俯仰角。 }\end{array}$

2) 规则描述:

$000 ! B Z p x d z *(($ Gama $<=-40 *$ Gama $>=-50$

) $\|($ Gama $>=40 *$ Gama $<=-50) \|$

TPGama2>1) $*$ Sita $>0 *$ Sita $<5 \quad 2$

0 盘旋类动作的起始标志

OPERATION BZpxdz=1

PRINT Z

3) 规则含义说明:

A.条件行 (000):

a) 000 ：事件号（可自定义）;

b) !BZpxdz*((Gama<=-40*Gama $>=-50) \quad \|$

(Gama $>=40 *$ Gama<=-50) \|| TPGama2 >1)

$*$ Sita $>0 * \operatorname{Sita}<5$ : 事件成立条件;

c) 2: 持续时间为 $1 \mathrm{~s}$;

d) 0: 事件性质;

e)盘旋类动作起始标志: 事件名称。

B. 操作行(OPERATION):

$\mathrm{BZpxdz}=1$ : 令盘旋类动作起始标志成 立。

\section{C.输出行(PRINT):}

$\mathrm{Z}$ : 不输出。

本规则中的 TPGana2 符号涉及倾斜角 变化量绝对值的计算, 这类不带附加条件的 参数计算也可以用规则进行描述:

000110 倾斜角变化量绝对值

OPERATION TPGama2=:\%Gama :

PRINT Z

其中, \%Gama ：表示按 $1 \mathrm{~s}$ 内的采样 点计算倾斜角变化量绝对值。

2. 计算坡度 $45^{\circ}$ 盘旋阶段飞行参数的 变化特征。

1) 符号定义:

TPPsi2: 航向角变化量绝对值;
SgmPI1：航向角累加变化量;

TPH2: 高度变化量绝对值;

SgmH3：高度累加变化量；

TPV2：速度变化量绝对值;

SgmV3: 速度变化量绝对值。

2) 规则描述:

000 BZpxdz 10 计算航向角累加变化量 OPERATION SgmPSI1=SgmPSI1+TPPsi 2

PRINT Z

000 BZpxdz 10 计算高度累加变化量

OPERATION SgmH3=SgmH3+TPH2

PRINT Z

000 BZpxdz 10 计算速度累加变化量 OPERATION SgmV3=SgmV3+TPV2

PRINT Z

3. 设立坡度 $45^{\circ}$ 盘旋完成标志

1) 符号定义:

BZ45px：坡度 $45^{\circ}$ 盘旋完成标志。

2) 规则描述:

000 SgmPSI $1>350 * \operatorname{SgmH} 3<500 * \mathrm{SgmV} 3<1501$

0 坡度 $45^{\circ}$ 盘旋完成标志成立

OPERATION BZ45px $=1$

PRINT Z

4. 动作识别

400 BZpxdz* BZ45px 10 坡度 $45^{\circ}$ 盘旋 OPERATION 0

PRINT 3, Gama (max), Gama (min)

PRINT Z

规则输出行含义说明:

3 : 输出类型, 代表输出动作完成的时 间范围;

Gama(max): 输出盘旋阶段的最大坡度 值;

Gama(min): 输出盘旋阶段的最小坡度 值。

其他飞行动作规则含义与以上实例相 同, 此处仅列出规则定义, 不再一一解释。

\subsection{3 组织方法}

知识的表达形式与推理机的推理策略 密切相关, 为了便于验证基于知识识别飞行 动作的有效性, 确定飞行动作识别推理机采 用数据驱动策略的正向精确推理, 因此知识 库整体结构组织的好坏非常重要。本论文采 用以下知识库结构组织方法。 
将不附带任何条件的参数预处理计算 规则放在知识库最前面; 不同类型的动作设 立各自的动作起始和完成标志, 避免规则之 间的相互干扰; 当判断出某个飞行动作已完 成, 或者根据飞行参数的变化判断出飞机已 处于平飞状态或飞机的运动已不属于该飞 行动作时, 立即将与该飞行动作有关的计算 参数和标志量清零, 为识别下一个飞行动作 做好准备; 建立与飞行动作识别知识库配套 的飞行参数译码数据库和计算参数说明数 据库。

采用上述知识表示方法具有以下优点: 充分利用了领域专家的先验知识; 整个识别 过程物理含义明确; 用户可以方便地根据实 际情况对知识库进行调整、扩充。知识库实 现时, 每一条规则采用XML格式记录。

\section{4 基于知识库的飞行动作识别}

飞行动作识别 ${ }^{[3,4]}$ 过程：逐帧读入飞行 数据, 推理机对知识库中的每一条规则进行 匹配, 当描述输出飞行动作的规则匹配成功 时, 根据知识库所指定的内容, 将飞行动作 的名称、完成时间和相关的飞行参数输出到 计算机屏幕和设立的数据文件中。

\section{3. 能力评价}

基于动作识别技术评价飞行能力是明 确飞行员每个飞行动作相对于有效动作的 飞行参数, 在多大程度上能够满足, 即明确 飞行效用的过程。最后给出测定对象关于该 项能力的效率判断。由于飞行动作的动态变 化, 使得判断需要考虑一定的模糊性、随机 性和不确定性, 采用传统的数学规划建模寻 求最优解或进行经验性决策, 常常难以满足 应用需求。本文引入模糊集理论 ${ }^{[5]}$, 以解决 含有模糊不确定因素的复杂决策问题, 结合 飞行任务的具体需求, 综合考虑多个参数不 同项目的要求, 根据专家评价标准和实测 值, 经过模糊变换后对被试作出评价。

根据飞行评估特点, 可主要采用三角形 和降半梯形综合模糊分布形式确定隶属函 数, 即假定每个评判因素对每个评判等级的 隶属函数是三角形分布或降半梯形分布。再 采用统计分析法, 通过分析大量训练样本, 将三角形模糊分布函数和降半梯形模糊分
布函数进行综合以后得到综合模糊分布隶 属函数（参见图1所示）:

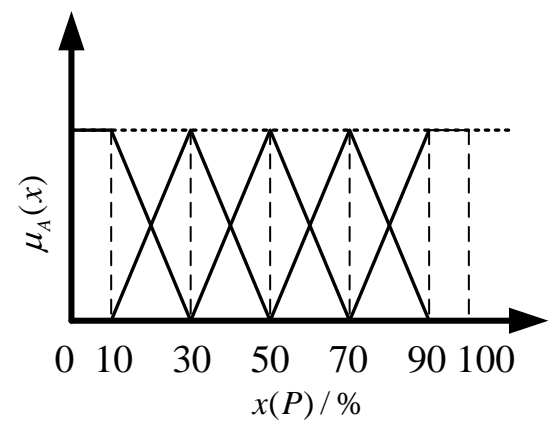

图1飞行能力综合评价隶属度函数

采用最小隶属度偏差法计算得出能力 排序。原理是推优者其各参数各项隶属度最 接近于理想方案。首先定义一个理想被试其 各项指标为理想解, 然后在实际资源集 $X$ 中 的有限个被试之间比较, 希望推优者越接近 于理想者则排序越靠前。定义理想资源 $x^{+}$ 的相对优属度向量为 $g=\left(g_{1}, g_{2}, \cdots, g_{m}\right)^{T}$, 其 中, $g_{i}=\max _{1 \leq j \leq n}\left\{\mu_{i}\right\} i=(\cdot \cdot \cdot, m 2$, 资源 $x_{j}\left(j=1,2 ; \cdot r\right.$ 与理想资源 $x^{+}$的接近程度 可用加权闵可夫斯基距离来刻画, $q$ 为距离 参数。

$$
d_{q}\left(x_{j}, x^{+}\right)=\left\{\sum_{i=1}^{m}\left[\omega_{i}\left(g_{i}-\mu_{i j}\right)\right]^{q}\right\}^{1 / q}
$$

\section{4. 结束语}

通过飞行动作识别技术记录管理并分 析飞行员实际操作能力, 从而构建飞行员选 拔、训练以及能力评价支撑平台, 并可进一 步为座舱、头盔等系统设计提供试验数据支 撑, 在工业研制和客户使用过程中均具有较 大的实际应用价值。

\section{References}

[1] Chunmei Gui, Qiang Jian, Architecture modeling for ergonomics of visual information based on software engineering, Software Engineering and Applications, vol. 2, pp. 93-97, 2013.

[2] Feiyue Huang, Guangyou Xu, Viewpoint independent action recognition, Journal of Software, vol. 7, pp. 1623-1634, 2008. 
[3] Yaqin $\mathrm{Hu}$, Movement recognition technology and its development, Ideo Engineering, vol. s2, pp. 451-453, 2013.

[4] Yongxiang Huang, Yi Hua, The design of the flight control system based on acceleration features action recognition extension, Intelligent Computer and Applications, vol. 2, pp. 46-49, 2012.
[5] Chaoqin Yin, Artificial intelligence approach and application, Wuhan: Huazhong university of science and technology press, 2007. 